Pacific Journal of Mathematics

AN EXTENSION OF SINGULAR HOMOLOGY TO BANACH 


\title{
AN EXTENSION OF SINGULAR HOMOLOGY TO BANACH ALGEBRAS
}

\author{
WILliam J. RALPH
}

\begin{abstract}
By making use of a simple connection with Banach algebras we introduce certain relations into singular homology and cohomology at the chain level and show that we obtain homology and cohomology theories. The deviation between singular and the new theory is measured by what turns out to be another homology theory $H M$. One of the main results is that $H M$ is zero on simplicial complexes but not on metric spaces in general. This shows that for any coefficient group there are an infinite number of different homology theories agreeing with the associated homology theory on simplicial complexes.
\end{abstract}

Section 2 shows that $H M$ detects all the anomalous singular homology constructed by Barratt and Milnor in [BM]. Section 3 gives a simple application to co-products and shows that we get the usual addition formula in homology for co-products without the assumption of a coidentity.

The main applications of this theory will be in a subsequent paper where the same relations are introduced into homotopy theory. The results of the present paper will show that the Hurewicz map factors through these new groups. Another application will be a nice (i.e. computable) way of relating the algebraic structure of $[X, H]$ ( $H$ an $H$-space) with properties of the maps induced by elements of $[X, H]$ in homology and cohomology.

1. In this section we will introduce a functor $A: \mathscr{T} \rightarrow \mathscr{C}$ where $\mathscr{T}$ is the category of spaces and continuous maps and $\mathscr{C}$ is the category of chain complexes of abelian groups and chain maps. This functor will be very similar to the functor $S$ which assigns to each space $X$ the singular complex $S X$ (see [G]).

For any two complex algebras $B_{1}$ and $B_{2}$ we defiine $L\left(B_{1}, B_{2}\right)$ to be the group of all complex linear maps from $B_{1}$ and $B_{2}$ under pointwise addition. Recall that for a space $X$, the group $S_{q} X$ in the singular chain complex $S X$ is the free abelian group on the set of all continuous maps from the $q$-simplex $\Delta_{q}$ into the space $X$. Every such map induces an algebra homomorphism from $C(X)$ to $C\left(\Delta_{q}\right)$, where $C(X)$ is the algebra 
of all complex valued continuous functions on $X$. This suggests that we might replace the group $S_{q} X$ by a group generated by certain algebra homomorphisms from $C(X)$ to $C\left(\Delta_{q}\right)$. However, we will not take the free group on such algebra homomorphisms but rather will use the natural group structure of $L\left(C(X), C\left(\Delta_{q}\right)\right)$.

(1) Definition. Let $X$ be any space. For $q \geq 0$ let $A_{q} X=\mathbf{Z}$-span of $\left\{\tau: C(X) \rightarrow C\left(\Delta_{q}\right) \mid \tau\right.$ is an algebra homomorphism induced by a continuous map $\left.\tau: \Delta_{q} \rightarrow X\right\} \subset L\left(C(X), C\left(\Delta_{q}\right)\right)$. For $q<0$ let $A_{q} X=0$.

The graded groups $S X$ and $A X$ are connected by the following:

(2) Definition. Let $\rho: S X \rightarrow A X$ be the homomorphism of graded groups defined on $S_{q} X$ as the unique homomorphism from $S_{q} X$ to $A_{q} X$ such that if $f \in C(X)$ and $\sigma: \Delta_{q} \rightarrow S$ is a generator of $S_{q} X$ then $\rho(\sigma)(f)=f \circ \sigma$, that is, $\rho(\sigma): C(X) \rightarrow C\left(\Delta_{q}\right)$ is the algebra homomorphism induced by $\sigma$.

It is easily shown that $A X$ can be made into a chain complex in a unique way by requiring that $\rho$ be a chain map. Similarly we can make $A$ into a covariant functor from $\mathscr{T}$ to $\mathscr{C}$, in a unique way, by requiring that $\rho$ be a natural transformation.

Let $\hat{\mathscr{A}}$ be the category of complex algebras with identity and identity preserving algebra homomorphisms. Let $\overline{\mathscr{A}}$ be the category of complex algebras and algebra homomorphisms. We can extend $A$, in a sense to be made clear, to functors $\hat{A}: \hat{\mathscr{A}} \rightarrow \mathscr{C}$ and $\bar{A}: \overline{\mathscr{A}} \rightarrow \mathscr{C}$ as follows:

(3) Definition. Let $B \in \hat{\mathscr{A}}$ be any algebra. Then for $q \geq 0$ let $\hat{A_{q}} B=\mathbf{Z}$-span of $\left\{\tau: B \rightarrow C\left(\Delta_{q}\right)\left|\tau: B \rightarrow C\left(\Delta_{q}\right)\right| \tau \in \mathscr{A}\right\} \subset L\left(B, C\left(\Delta_{q}\right)\right)$ and for $q<0$ let $\hat{A}_{q} B=0$.

We make a similar definition for $\bar{A}_{q} B$ and $B \in \overline{\mathscr{A}}$. The graded groups $\hat{A B}$ and $\overline{A B}$ can be made into chain complexes by defining a boundary operator as follows. We define $\partial(\tau)=\rho\left(\partial_{\mathrm{id}_{q}}\right) \circ \tau$, where id ${ }_{q}: \Delta_{q} \rightarrow \Delta_{q}$ is the identity map considered as an element of $S_{q}\left(\Delta_{q}\right)$. With this definition we obtain functors $\bar{A}: \overline{\mathscr{A}} \rightarrow \mathscr{C}$ and $\hat{A}: \hat{\mathscr{A}} \rightarrow \mathscr{C}$. We now make clear the sense in which $\hat{A}$ is an extension of $A$.

(4) Proposition. Let $\mathscr{T}^{\prime}$ be the full subcategory of $\mathscr{T}$ whose objects are compact Hausdorff spaces. Then

$$
\left.\hat{\mathscr{A}} \circ C\right|_{\mathscr{T}^{\prime}}=\left.A\right|_{\mathscr{T}^{\prime}} .
$$


Proof. This follows immediately from the well known fact that any algebra homomorphism from $C(X)$ to $C(Y)$, where $X$ and $Y$ are compact Hausdorff, is induced by a continuous map from $Y$ to $X$.

Now let $\mathscr{B}$ be the category of commutative Banach algebras with identity and $G: \mathscr{B} \rightarrow \mathscr{T}$ the Gelfand factor. The previous proposition suggests the following:

(5) THEOREM ( see $[\mathbf{R}])$. There exists a natural chain isomorphism from $\hat{\left.A\right|_{\mathscr{B}}}$ to $\left.A \circ G\right|_{\mathscr{B}}$.

We can also show

(6) Proposition (see [R]). Let $M$ be a maximal ideal in some algebra $B \in \mathscr{B}$. Then

$$
H_{q} \hat{A B} \cong H_{q} \bar{A} M \quad \text { for all } q>0
$$

and

$$
H_{0} \hat{A B} \cong H_{0} \bar{A} M \oplus \mathbf{Z}
$$

(7) Definition. Let $M: \mathscr{T} \rightarrow \mathscr{C}$ be the functor that is the kernel of the natural transformation $\rho$ (see (2)).

There is a short exact sequence $0 \rightarrow M X \rightarrow S X \rightarrow A X \rightarrow 0$ of chain complexes for any space $X$. We turn now to the characterization of elements in $M X$.

(8) Definition. For any collection of continuous maps $\theta_{i}: X \rightarrow Y$, $i \in I$ we let $I(x, y)=\left\{i \in I \mid \theta_{i}(x)=y\right\}$.

(9) Proposition. Let $X$ be an arbitrary topological space and let $Y$ be any metric space. Let $\theta_{i}: X \rightarrow Y, i \in I$, be any finite set of not necessarily distinct continuous maps. Denote by $\tau_{i}$ the algebra homomorphism induced by $\boldsymbol{\theta}_{i}$ from $C(Y)$ to $C(X)$. If $\left\{v_{l}\right\}_{i \in I} \subset \mathbf{Z}$ then:

$$
\sum_{I} v_{i} \tau_{l}=0 \text { in } L(C(Y), C(X)) \Leftrightarrow \sum_{I(x, y)} v_{\imath}=0 \text { for all }(x, y) \in X \times Y .
$$

(Note: when $I(x, y)$ is empty we define $\Sigma_{I(x, y)} v_{i}=0$.) 
Proof. $\Rightarrow$ Fix $(x, y) \in X \times Y$ and assume $I(x, y) \neq \varnothing$. Define $Y_{x}=$ $\left\{z \in Y \mid \theta_{i}(x)=z\right.$ for some $\left.i \in I\right\}$. Now let $f$ be a continuous function such that $f(y)=1$ and $f$ vanishes on any elements in $Y_{x} \backslash\{y\}$. Evaluating $\sum_{I} v_{t} \tau_{i}$ at $f$ and then at $x$ we have

$$
0=\left(\sum_{I} v_{i} \tau_{i}\right)(f)(x)=\sum_{I} v_{i} f \circ \theta_{i}(x)=\sum_{I(x, y)} v_{i}
$$

by our choice of $f$.

$\Leftarrow$ Let $f \in C(Y)$ and $x \in X$, then

$$
\left(\sum_{I} v_{i} \tau_{\iota}\right)(f)(x)=\sum_{I} v_{i} f \circ \theta_{i}(x)=\sum_{y \in Y_{x}}\left(f(y) \cdot \sum_{I(x, y)} v_{i}\right)=0 .
$$

Corollary. Let $X$ be a metric space. If $\sum_{I} v_{i} \sigma_{t} \in S_{q} X$ then

$$
\rho\left(\sum_{I} v_{\imath} \sigma_{i}\right)=0 \Leftrightarrow \sum_{I(x, y)} v_{\imath}=0 \quad \text { for all }(t, x) \in \Delta_{q} \times X .
$$

It can be shown that $\rho$ is injective when restricted to the subgroup of $S_{q} X$ generated by simplicial maps. This suggests a result we will shortly prove, namely that for a simplicial complex $X, H_{*} M X=0$ and hence $H_{*} X \cong H_{*} A X$.

Corollary (10) suggests the analogues of the complexes $M X$ and $A X$ when coefficients are taken in some group $G$.

(11) DEFINITION:

$$
\begin{gathered}
M_{q}(X \mid G)=\left\{\sum_{I} g_{i} \sigma_{i} \subset S_{q} X \otimes G \mid \sum_{I(t, x)} g_{i}=0 \text { for all }(t, x) \in \Delta_{q} \times X\right\} \\
A_{q}(X \mid G)=S_{q} X \otimes G / M_{q}(X \mid G)
\end{gathered}
$$

Note that $M_{q}(X \mid \mathbf{Z}) \cong M_{q}(X)$ and $A_{q}(X \mid \mathbf{Z}) \cong A_{q}(X)$. We will let $M(\mid G)$ and $A(\mid G)$ denote the obvious functors from $\mathscr{T}$ to $\mathscr{C}$. We now set out to show that $M(\mid G)$ and $A(\mid G)$ are homology theories on $\mathscr{T}$. There are short exact sequences

$$
0 \rightarrow A(Y \mid G) \rightarrow A(X \mid G) \rightarrow A(X \mid G) / A(Y \mid G) \rightarrow 0
$$

for any spaces $Y \subset X$. We define $M(X, Y \mid G)=M(X \mid G) / M(Y \mid G)$ and $A(X, Y \mid G)=A(X \mid G) / A(Y \mid G)$.

(12) TheOREM ( Homotopy Invariance). Let $(X, Y)$ and $(W, Z)$ be any pairs of spaces. If $f_{0}, f_{1}:(X, Y) \rightarrow(W, Z)$ are homotopic, then

$$
H M\left(f_{0} \mid G\right)=H M\left(f_{1} \mid G\right): H M(X, Y \mid G) \rightarrow H M(W, Z \mid G)
$$


and

$$
H A\left(f_{0} \mid G\right)=H A\left(f_{1} \mid G\right): H A(X, Y \mid G) \rightarrow H A(W, Z \mid G) .
$$

This theorem is proved in singular homology by defining an operator $P$ called the prism operator (see $[\mathbf{G}]$ ). The proof of Theorem (12) proceeds identically once we have shown that the prism operator on $S X \otimes G$ can be suitably restricted to $M(X \mid G)$. This is contained in the following which uses nothing more than the naturality of $P$.

(13) Lemma. Let $P$ be the prism operator on $S X \otimes G$. Then $P\left(M_{q}(X \mid G) \subset M_{q+1}(X \times I \mid G)\right)$.

Proof. Let $\mathrm{id}_{q} \in S_{q}\left(\Delta_{q}\right)$ be the identity map. Suppose $P\left(\mathrm{id}_{q}\right)=$ $\sum_{j \in J} w_{j} \delta_{j}$ where $w_{j} \in \mathbf{Z}$ and $\delta_{j} \in S_{q+1}\left(\Delta_{q} \times I\right)$. Let $\sum_{I} g_{i} \sigma_{i} \in M_{q}(X \mid G)$. By the naturality of the prism map we have

$$
\begin{aligned}
P\left(\sum_{I} g_{i} \sigma_{i}\right) & =\sum_{I} g_{i} P\left(\sigma_{i}\right)=\sum_{I} g_{i} S_{q+1}\left(\sigma_{i} \times \mathrm{id}\right)\left(\sum_{J} w_{j} \delta_{j}\right) \\
& =\sum_{J} w_{j} \sum_{I} g_{i} S_{q+1}\left(\sigma_{i} \times \mathrm{id}\right) \circ \delta_{j} .
\end{aligned}
$$

Let $f_{i}^{j}=S_{q+1}\left(\sigma_{i} \times \mathrm{id}\right) \circ \delta_{j}$. We will show that $\sum_{I} g_{i} f_{i}^{j} \in M_{q+1}(X \times I \mid G)$. Let $t \in \Delta_{q+1}$ and $(a, b) \in X \times I$. Then

$$
\begin{aligned}
I(t,(a, b)) & =\left\{i \mid S_{q+1}\left(\sigma_{i} \times \mathrm{id}\right) \circ \delta_{j}(t)=(a, b)\right\} \\
& =\left\{i \|\left(\sigma_{i}\left(\delta_{j}^{1}(t)\right), \delta_{j}^{2}(t)\right)=(a, b)\right\}=\varnothing \text { or }\left\{i \mid \sigma_{i}\left(\delta_{j}^{1}(t)\right)=a\right\} .
\end{aligned}
$$

Therefore

$$
\sum_{I(t,(a, b))} g_{i}=\sum_{\left\{i \mid \sigma_{i}\left(\delta_{J}^{1}(t)\right)=a\right\}} g_{t}=0 \quad \text { since } \sum_{I} g_{i} \sigma_{i} \in M(X \mid G)
$$

or

$$
\sum_{\varnothing} g_{i}=0
$$

and hence

$$
P\left(\sum_{I} g_{i} \sigma_{i}\right) \in M_{q+1}(X \times I \mid G)
$$

(14) THEOREM (Excision). Let $(X, Y)$ be any metric pair. If $U$ is any open subset of $X$ such that $\bar{U} \subset$ int $Y$, then the excision map $J:(X-U$, $Y-U) \rightarrow(X, Y)$ induces isomorphisms

$$
H M(J \mid G): H M(X-U, Y-U \mid G) \rightarrow H M(X, Y \mid G)
$$


and

$$
H A(J \mid G): H A(X-U, Y-U \mid G) \rightarrow H A(X, Y \mid G) .
$$

We will first prove excision for $H M$. Excision for $H A$ will then follow from the five lemma. In outline the proof of excision for $H M$ is the same as that for singular homology (see [G]). The only substantial difference lies in the proof of Lemma (16), which requires a new idea.

The subdivision operator Sd: $S_{q}(X) \otimes G \rightarrow S_{q}(X) \otimes G$ and the degeneracy operator $T: S_{q}(X) \otimes G \rightarrow S_{q+1}(X) \otimes G$ can be suitably restricted to $M_{q}(X \mid G)$ with their usual properties. The proof of this is the same as that for the prism operator and uses nothing more than the naturality of $\mathrm{Sd}$ and $T$.

(15) Lemma. Let $X$ be any space with $Y \subset X$. If $z \in M_{q}(X \mid G)$ and $\partial z \in M_{q-1}(Y \mid G)$, then, for all $n, z-\operatorname{Sd}^{n} z \in \partial M_{q+1}(X \mid G)+M_{q}(Y \mid G)$.

Proof. Identical to (15.14) of [G].

(16) Lemma. Let $X$ be a metric space with $z \in M_{q}(X \mid G)$ and $\mathscr{U}$ an open cover of $X$. Then there exists an $r$ so that $\operatorname{Sd}^{r} z=\Sigma_{L} z_{l}$ with $z_{l} \in$ $M_{q}\left(U_{l} \mid G\right)$ and $\left\{U_{l}\right\}_{l \in L} \subset \mathscr{U}$.

Proof. Fix $z=\sum_{j=1}^{n} g_{l} \sigma_{i}$ where $g_{i} \in G$ and $\sigma_{i}: \Delta_{q} \rightarrow X$. Let id ${ }_{q}$ : $\Delta_{q} \rightarrow \Delta_{q}$ be the identity map and suppose that $\operatorname{Sd}\left(\mathrm{id}_{q}\right)=\sum_{J=1}^{n} w_{J} \delta_{J}$ for integers $w_{j}$ and maps $\delta_{j}: \Delta_{q} \rightarrow \Delta_{q}$. An easy induction argument shows that

$$
\mathrm{Sd}^{r} z=\sum_{J_{1}, \ldots, j_{r}=1}^{m} w_{j_{1}} \cdots w_{J_{r}}\left(\sum_{i=1}^{n} g_{l} \sigma_{i} \circ \delta_{j_{1}} \circ \cdots \circ \delta_{J_{r}}\right) .
$$

Let $W \in X$ be the union of the images of all the $\sigma_{i} . W$ is compact since it is the finite union of compact sets. Now apply the Lebesgue covering lemma to the space $W$ and the open cover $\mathscr{V}$ of $W$ defined by $\mathscr{V}=\{U \cap W \mid U \in \mathscr{U}\}$. This yields an $\varepsilon>0$ such that if $Z \subset W$ with $\operatorname{diam}(Z)<\varepsilon$ then there is a $V \in \mathscr{V}$ with $Z \subset V$. By (15.13) of [G] we may choose $r$ so that each of the maps $\sigma_{i} \circ \delta_{j_{1}} \circ \cdots \circ \delta_{j_{r}}$ is such that $\operatorname{diam}\left(\operatorname{im} \sigma_{l} \circ \delta_{j_{1}} \circ \cdots \circ \delta_{j_{r}}\right)<\varepsilon / n$. This $r$ will be fixed for the remainder of the proof. 
For any integers $1 \leq j_{1}, \ldots, j_{r} \leq m$, the set

$$
W\left(j_{1}, \ldots, j_{r}\right)=\bigcup_{i=1}^{m} \operatorname{im} \sigma_{i} \circ \delta_{J_{1}} \circ \cdots \circ \delta_{J_{r}}
$$

has a finite number of compact components since it is a finite union of compact connected sets. Define

$$
\begin{array}{r}
D\left(j_{1}, \ldots, j_{r}\right)=\left\{A \subset\{1, \ldots, n\} \mid \exists \text { a component, } C \text {, of } W\left(j_{1}, \ldots, j_{r}\right)\right. \\
\text { with } \left.A=\left\{i \in\{1, \ldots, n\} \text { limage of } \sigma_{\imath} \circ \delta_{j_{1}} \circ \ldots \circ \delta_{j_{r}} \subset C\right\}\right\} .
\end{array}
$$

Since $W\left(j_{1}, \ldots, j_{r}\right)$ is the disjoint union of its components it follows that $D\left(j_{1}, \ldots, j_{r}\right)$ is a partition of $\{1, \ldots, n\}$.

We now show that if $A \in D\left(j_{1}, \ldots, j_{r}\right)$ then

$$
\sum_{i \in A} g_{i} \sigma_{i} \circ \delta_{j_{1}} \circ \cdots \circ \delta_{j_{r}} \in M_{q}(U \mid G) \text { for some } U \in \mathscr{U} \text {. }
$$

First we show that $\sum_{i \in A} g_{i} \sigma_{i} \circ \delta_{j_{1}} \circ \ldots \circ \delta_{j_{r}} \in M_{q}(X \mid G)$ and then that $\bigcup_{i \in A} \operatorname{im} \sigma_{i} \circ \delta_{j_{1}} \circ \ldots \circ \delta_{j_{r}} \subset U$ for some $U \in \mathscr{U}$.

Let $C$ be the component of $W\left(j_{1}, \ldots, j_{r}\right)$ associated with $A$ and suppose $x \in C$ and $t \in \Delta_{q}$. Since $\sum_{i=1}^{\infty} g_{i} \sigma_{i} \in M_{q}(X \mid G)$ we must have $\sum_{I\left(\delta_{j 1} \circ \cdots \circ \delta_{j t}(t), x\right)} g_{i}=0$. But this implies that $\sum_{i \in A} g_{l}=0$ since only those maps $\sigma_{i} \circ \delta_{j_{1}} \circ \cdots \circ \delta_{j_{r}}$ with $i \in A$ can have image containing $x$. We conclude that $\sum_{i \in A} g_{l} \sigma_{i} \circ \delta_{j_{1}} \circ \cdots \circ \delta_{j_{r}} \in M_{q}(X \mid G)$. Since $C$ is connected and $\operatorname{diam}\left(\operatorname{im} \sigma_{i} \circ \delta_{J_{1}} \circ \cdots \circ \delta_{j_{r}}\right)<\varepsilon / n$, it follows from the triangle inequality that $\operatorname{diam}(C)<\operatorname{card}(A) \cdot \varepsilon / n \leq n \cdot \varepsilon / n=\varepsilon$. By our choice of $\varepsilon$ there must be $V \in \mathscr{V}$ with $C \subset V$. But $V$ is of the form $U \cap W$ for some $U \in \mathscr{U}$ and hence there is $U \in \mathscr{U}$ with $C \subset U$. Therefore $\sum_{i \in A} g_{i} \sigma_{t} \circ \delta_{j_{1}} \circ \cdots \circ \delta_{j_{r}} \in M_{q}(U \mid G)$ for some $U \in \mathscr{U}$.

We can now complete the proof of the lemma as follows:

$$
\begin{aligned}
\operatorname{Sd}^{r} z & =\sum_{j_{1}, \ldots, j_{r}=1}^{m} w_{j_{1}} \cdots w_{j_{r}}\left(\sum_{i=1}^{n} g_{i} \sigma_{i} \circ \delta_{j_{1}} \circ \cdots \circ \delta_{j_{r}}\right) \\
& =\sum_{j_{1}, \ldots, j_{r}=1}^{m} w_{j_{1}} \cdots w_{j_{r}}\left(\sum_{A \in D\left(j_{1} \cdots j_{r}\right)}\left(\sum_{i \in A} g_{i} \sigma_{l} \circ \delta_{j_{1}} \circ \cdots \circ \delta_{j_{r}}\right)\right) \\
& \left(\text { since } D\left(j_{1}, \ldots, j_{r}\right) \text { is a partition of }\{1, \ldots, n\}\right) \\
= & \sum_{j_{1}, \ldots, j_{r}}^{m} \sum_{A \in D\left(j_{1}, \ldots, j_{r}\right)} w_{j_{1}} \cdots w_{j_{r}}\left(\sum_{i \in A} g_{i} \sigma_{i} \circ \delta_{j_{1}} \circ \cdots \circ \delta_{j_{r}}\right) .
\end{aligned}
$$

Since

$$
w_{j_{1}} \cdots w_{j_{r}} \cdot\left(\sum_{i \in A} g_{i} \sigma_{i} \circ \delta_{j_{1}} \circ \cdots \circ \delta_{j_{r}}\right) \in M_{q}(U \mid G)
$$


for some $U \in \mathscr{U}$ we see that $\operatorname{Sd}^{r} z=\sum_{L} z_{l}$ and $U_{l} \in \mathscr{U}$.

With Lemma (16) in hand the proof of Theorem (14) for $H M$ follows identically to that given in [G]. Excision for $H A$ now follows from excision for $H M$ and $H S$ and the five lemma applied to the diagram of long exact sequences obtained from the diagram

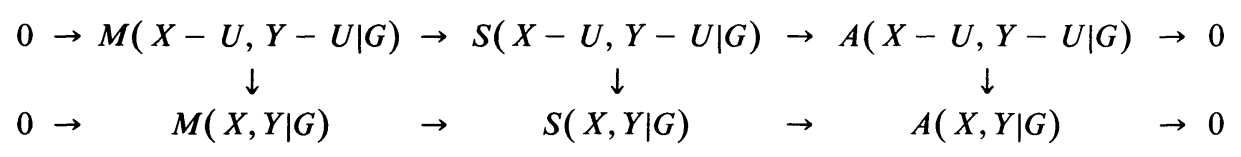

Technical Remark. We have been unable to prove excision directly for $H A$. This was the original reason for introducing $M$.

In summary we have proved:

(17) TheOREM. $H M(, \mid G)$ and $H A(, \mid G)$ are homology theories on the category of pairs of metric spaces.

On simplicial complexes we can identify exactly what our functors are.

(18) THEOREM. If $(X, Y)$ is any pair of spaces homotopy equivalent to a pair of simplicial complexes (not necessarily finite), then $H M(X, Y \mid G)=0$ and $H A(X, Y \mid G) \cong H S(X, Y \mid G)$ (naturally).

Proof. $H M($ point $\mid G)=0$. Therefore by the uniqueness theorem $H M$ vanishes on finite simplicial complexes. It is easily checked that $H M$ is a homology theory with "compact carriers" (see 4.8 .11 of [S]) and hence the first result follows. The second result is immediate from the long exact sequence relating $H M, H S$ and $H A$.

Cohomology. Using results of Bergman [B] it is easy to move that $A(X)$ is a free abelian group. In $[\mathbf{R}]$ we give a long and tortuous proof that $A(X) / A(Y)$ is free abelian for $Y$ an open subset of a metric space $X$. This is enough to prove the analogue of Theorem (18) in cohomology for $G=\mathbf{Z}$. We mention in passing the following interesting connection with the first Čech cohomology group $\check{H}^{1}(X ; \mathbf{Z})$.

(19) THEOREM (see [R]). If $X$ is a locally path connected metric space then there is a natural isomorphism $\eta: \check{H}^{1}(X) \rightarrow H^{1} A(X)$. 
2. In this section we will show that $H_{*} M$ is non-trivial on the following spaces:

(20) Definition. Let $X_{(r)}$ be a countable union of $r$ spheres having a single point in common and a metric topology in which the diameter of the spheres tends to zero with increasing index.

Our proof that $H_{*} M\left(X_{(r)}\right) \neq 0$ will be based on a result of Barratt and Milnor, namely, that the singular homology of $X_{(r)}$ (for $r \geq 2$ ) is anomalous in the sense that $H_{q} S\left(X_{(r)}\right) \neq 0$ for arbitrarily large $q$. We will see that there is a one-one correspondence between the elements they construct and certain elements of $H_{*} M\left(X_{(r)}\right)$. Their theorem is the following:

(21) THEOREM (see $[\mathbf{B M}]$ ). The rational singular homology groups $H_{q} S\left(X_{(r)} ; Q\right)$ with $q \equiv 1 \bmod (r-1), q>1, r>1$ are not zero. In fact these groups are not even countable.

Their proof is based upon the Hurewicz homomorphism $\omega_{Q}: \pi_{q}\left(X_{(r)}\right)$ $\rightarrow H_{q} S\left(X_{(r)} ; Q\right)$. The non-trivial elements they construct, are images under $\omega_{Q}$ of elements in $\pi_{q}\left(X_{(r)}\right)$ built up from Whitehead products. The application of (10) requires that we have descriptions of maps at the point set level. For this reason, in the following definition of Whitehead products and other subsequent definitions, we will be careful to make all choices of representatives explicit.

(22) Definition (Whitehead Product). Let $I^{n}$ be the oriented $n$-cube. Let $\alpha \in \pi_{m+1}(X, *)$ and $\beta \in \pi_{n+1}(X, *)$ have representatives $f$ : $\left(I^{m+1}, \partial I^{m+1}\right) \rightarrow(X, *)$ and $g:\left(I^{n+1}, \partial I^{n+1}\right) \rightarrow(X, *)$ respectively. The boundary of $I^{m+n+2}$ equals $\partial\left(I^{m+1} \times I^{n+1}\right)=I^{m+1} \times \partial I^{n+1} \cup \partial I^{m+1} \times$ $I^{n+1}$ which is an $m+n+1$ sphere, $S$, oriented by the usual orientation on $I^{m+n+2}$. We define a map $e(f, g):(X, *) \rightarrow(X, *)$ by

$$
e(f, g)(x, y)= \begin{cases}f(x) & (x, y) \in I^{m+1} \times \partial I^{n+1}, \\ g(x), & (x, y) \in \partial I^{m+1} \times I^{n+1}\end{cases}
$$

Now fix for all time an orientation preserving homeomorphism $\lambda_{m+n+1}$ : $\left(I^{m+n+1}, \partial I^{m+n+1}\right) \rightarrow\left(\partial I^{m+n+2}, *\right)$. Denote by $\{f, g\}$ the composition $e(f, g) \circ \lambda$. This is the representative of an element in $\pi_{m+n+1}(X, *)$ which is called the Whitehead product of $\alpha$ and $\beta$ and denoted $[\alpha, \beta]$ or $[\{f, g\}]$ when we wish to make the choice of representative explicit. 
Basic properties of the Whitehead product can be found in [W].

The next two definitions are in preparation for the description of the elements constructed by Barratt and Milnor.

(24) Definition. By an infinite sum of elements $\left[h_{j}\right] \in \pi_{q}(X, *)$, $j=1,2, \ldots$, we will mean an element $[h] \in \pi_{q}(X, *)$, where $h:\left(I^{q}, \partial I^{q}\right)$ $\rightarrow(X, *)$ is given by

$$
\begin{aligned}
& h\left(t_{1}, \ldots, t_{q}\right) \\
& =h_{j}\left(\left(t_{1}-\left(1-\frac{1}{2^{j-1}}\right)\right) /\left(1-\frac{1}{2^{j}}\right)-\left(1-\frac{1}{2^{j-1}}\right), t_{2}, \ldots, t_{q}\right) \\
& \quad \text { for } t_{1} \in\left[1-\frac{1}{2^{j-1}}, 1-\frac{1}{2^{J}}\right] .
\end{aligned}
$$

Note that part of this definition is that the $h_{j}$ must be such that $h$ is continuous. We will denote $h$ by $\sum_{j=1}^{\infty} h_{j}$.

(26) Definition. We will say that $[h] \in \pi_{q}(X, *)$ is an infinite sum of Whitehead products if $h=\sum_{J=1}^{\infty}\left\{f_{J}, g_{J}\right\}$, where $f_{J}$ and $g_{j}$ are representatives of elements in $\pi_{m_{j}+1}(X, *)$ and $\pi_{n_{j}+1}(X, *)$, respectively, with $m_{J}+$ $n_{j}+1=q$. We will denote an infinite sum of Whitehead products by $\left[\sum_{j=1}^{\infty}\left\{f_{j}, g_{j}\right\}\right]$.

(27) Proposition. All of the elements of $H_{q} S\left(X_{(r)}, *\right)$, with $q \equiv$ $\bmod (r-1), q>r, \quad r>1$, constructed in [BM], are of the form $\omega_{Q}\left(\left[\sum_{j=1}^{\infty}\left\{f_{j}, g_{j}\right\}\right]\right)$.

We will require a point set description of the Hurewicz map.

(28) Definition. Fix for all time an orientation preserving homeomorphism $l:\left(\Delta_{q}, \partial \Delta_{q}\right) \rightarrow\left(I^{q}, \partial I^{q}\right)$. Let $C_{q}$ be the constant map $\left(\Delta_{q}, \Delta_{q}\right)$ $\rightarrow(X, *)$. Then for $[f] \in \pi_{q}(X, *)$, the Hurewicz map $\pi: \pi_{q}(X, *) \rightarrow$ $H_{q} S(X)$ can be described as

$$
\omega([f])= \begin{cases}{[f \circ l]} & \text { if } q \text { is odd } \\ {\left[f \circ l-C_{q}\right]} & \text { if } q \text { is even. }\end{cases}
$$

(30) Definition. For $[f] \in \pi_{k}(X, *)$, we define $\eta(f)$ to be the constant map $\left(I^{k}, I^{k}\right) \rightarrow(X, *)$.

The elements in the next lemma will be used to construct non-trivial elements in $H_{*} M\left(X_{(r)}\right)$. 
(31) Lemma. Let $\left[\sum_{j=1}^{\infty}\left\{f_{j}, g_{j}\right\}\right] \in \pi_{q}(X, *)$. Then

(i) $\sum_{j=1}^{\infty}\left\{\eta\left(f_{j}\right), g_{j}\right\}$ and $\sum_{j=1}^{\infty}\left\{f_{j}, \eta\left(g_{j}\right)\right\}$ are continuous;

(ii) $\left[\sum_{j=1}^{\infty}\left\{\eta\left(f_{j}\right), g_{J}\right\}\right]=\left[\sum_{j=1}^{\infty}\left\{f_{j}, \eta\left(f_{j}\right)\right\}\right]=0$ in $\pi_{q}(X, *)$.

Proof. (i) obvious

(ii) We will show that $\left[\sum_{j=1}^{\infty}\left\{\eta\left(f_{j}\right), g_{j}\right\}\right]=0$, the proof for the other term being identical. Since image $\eta\left(f_{j}\right)=*$, we can regard $\left[\left\{\eta\left(f_{j}\right), g_{j}\right\}\right]$ as an element of $\pi_{q}\left(\right.$ image $\left.g_{J}, *\right)$ by $(23)$. $\left[\left\{\eta\left(f_{j}\right), g_{J}\right\}\right]=0$ in $\pi_{q}$ (image $g_{J}, *$ ) (since the Whitehead product of anything with the trivial element is zero) and hence there is a homotopy $H_{j}: I \times\left(I^{q}, \partial I^{q}\right) \rightarrow\left(\right.$ image $\left.g_{j}, *\right)$ from $\left\{\eta\left(f_{j}\right), g_{j}\right\}$ to the constant map. Since the image of $H_{j}$ is contained in the image of $g_{j}$, these homotopies can be glued together in the obvious way to form a homotopy from $\sum_{j=1}^{\infty}\left\{\eta\left(f_{j}\right), g_{j}\right\}$ to the trivial map.

(32) Proposition. If $\left[\sum_{j=1}^{\infty}\left\{f_{j}, g_{j}\right\}\right] \in \pi_{q}(X, *)$ is an infinite sum of Whitehead products, then there exists $[\psi] \in H_{q} M(X)$ such that $H_{q}(i)[\psi]$ $=\omega\left(\left[\sum_{j=1}^{\infty}\left\{f_{j}, g_{j}\right\}\right]\right)$ where $i: M(X) \rightarrow S(X)$ is the inclusion.

Proof. Using Lemma (31) and (29) we have

$$
\begin{aligned}
\left(\left[\sum_{j=1}^{\infty}\left\{f_{j}, g_{j}\right\}\right]\right) \\
=\omega\left(\left[\sum_{j=1}^{\infty}\left\{f_{j}, g_{j}\right\}\right]\right)-\omega\left(\left[\sum_{j=1}^{\infty}\left\{\eta\left(f_{J}\right), g_{J}\right\}\right]\right) \\
\quad-\omega\left(\left[\sum_{j=1}^{\infty}\left\{f_{J}, \eta\left(f_{J}\right)\right\}\right]\right)+\omega\left(\left[\sum_{j=1}^{\infty}\left\{\eta\left(f_{j}\right), \eta\left(g_{j}\right)\right\}\right]\right) \\
\left.=\left[\left(\sum_{j=1}^{\infty}\left\{f_{j}, g_{j}\right\}\right) \circ l-\left(\sum_{j=1}^{\infty}\left\{\eta\left(f_{j}\right), g_{j}\right\}\right) \circ l\right]\right) \\
\left.\quad-\left(\sum_{j=1}^{\infty}\left\{f_{j}, \eta\left(g_{j}\right)\right\}\right) \circ l-\left(\sum_{j=1}^{\infty}\left\{\eta\left(f_{j}\right), \eta\left(g_{j}\right)\right\}\right) \circ l\right] \\
=[\psi] \text { (say). }
\end{aligned}
$$

Note that (33) will hold even if $q$ is even since the $C_{q}$ terms will all cancel out (see (28)). We will complete the proof of the proposition by using the criterion given in (10) to show that $\psi \in M_{q}(X)$. 
Let $u \in \Delta_{q}$. Then

$$
\begin{aligned}
& \left(\sum_{j=1}^{\infty}\left\{f_{j}, g_{j}\right\}\right) \circ l(u)=\left(\sum_{j=1}^{\infty}\left\{f_{j}, g_{j}\right\}\right)\left(\bar{t}_{1}, \ldots, \bar{t}_{q}\right) \text { for some } \\
\qquad\left(\bar{t}_{1}, \ldots, \bar{t}_{q}\right) \in I^{q} \text { with } \bar{t}_{1} \in\left[1-\frac{1}{2^{k-1}}, 1-\frac{1}{2^{k}}\right], & \quad\left\{f_{j}, g_{j}\right\}\left(\left(\bar{t}_{1}-\left(1-\frac{1}{2^{k-1}}\right)\right) /\left(1-\frac{1}{2^{k}}\right)-\left(1-\frac{1}{2^{k-1}}\right), \bar{t}_{2}, \ldots, \bar{t}_{q}\right) \\
= & e\left(f_{j}, g_{j}\right) \\
& \circ \lambda\left(\left(\bar{t}_{1}-\left(1-\frac{1}{2^{k-1}}\right)\right) /\left(1-\frac{1}{2^{k}}\right)-\left(1-\frac{1}{2^{k-1}}\right), \bar{t}_{2}, \ldots, \bar{t}_{q}\right) \\
= & e\left(f_{j}, g_{j}\right)(x, y) \text { by (22). and (25), }
\end{aligned}
$$

which we think of as being $I^{m_{k}+1} \times \partial I^{n_{k}+1} \cup \partial I^{m_{k}+1} \times I^{n_{k}+1}$, and where $\left[f_{k}\right] \in \pi_{m_{k}+1}(X, *)$ and $\left[g_{k}\right] \in \pi_{n_{k}+1}(X, *)(\operatorname{see}(22)$ and (23)).

Similarly we obtain

$$
\begin{array}{r}
\left(\sum_{j=1}^{\infty}\left\{\eta\left(f_{j}\right), g_{j}\right\}\right) \circ l(u)=e\left(\eta\left(f_{k}\right), g_{k}\right)(x, y) \\
\left(\sum_{j=1}^{\infty}\left\{f_{j}, \eta\left(g_{j}\right)\right\}\right) \circ l(u)=e\left(f_{k}, \eta\left(g_{k}\right)\right)(x, y)
\end{array}
$$

$$
\left(\sum_{j=1}^{\infty}\left\{\eta\left(f_{j}\right), \eta\left(g_{j}\right)\right\}\right) \circ l(u)=e\left(\eta\left(f_{k}\right), \eta\left(g_{k}\right)\right)(x, y) .
$$

There are two cases to consider. If $(x, y) \in I^{m_{k}+1} \times \partial I^{n_{k}+1}$ then by (23) we have:
(i) $e\left(f_{k}, g_{k}\right)(x, y)=f_{k}(x)$
(ii) $e\left(\eta\left(f_{k}\right), g_{k}\right)(x, y)=*$
(iii) $e\left(f_{k}, \eta\left(g_{k}\right)\right)(x, y)=f_{k}(x)$
(iv) $e\left(\eta\left(f_{k}\right), \eta\left(g_{k}\right)\right)(x, y)=*$.

Similarly if $(x, y) \in \partial I^{m_{k}+1} \times I^{n_{k}+1}$ we have:
(i) $e\left(f_{k}, g_{k}\right)(x, y)=g_{k}(y)$
(ii) $e\left(\eta\left(f_{k}\right), g_{k}\right)(x, y)=g_{k}(y)$
(iii) $e\left(f_{k}, \eta\left(g_{k}\right)\right)(x, y)=*$
(iv) $e\left(\eta\left(f_{k}\right), \eta\left(g_{k}\right)\right)(x, y)=*$. 
If we let $v_{i}$ be the set of coefficients of the four terms in (33) we see that $\sum_{i(u, z)} v_{i}=0$ for any $z \in X$. Since $u$ was arbitrary, we conclude, by (10), that $\psi \in M_{q}(X)$.

(38) THEOREM. $H_{q} M\left(X_{(r)}, Q\right)$ and $H_{q} M\left(X_{(r)}\right)$ are uncountable groups for $q \equiv 1 \bmod (r-1), q>r, r>1$.

Proof. Suppose $q \equiv 1 \bmod (r-1), q>r, r>1$. Then, by (27), there are an uncountable number of elements in $H_{q} S\left(X_{(r)}, Q\right)$ of the form $\omega_{Q}\left(\left[\sum_{j=1}^{\infty}\left\{f_{j}, g_{j}\right\}\right]\right)$, where we recall that $\omega_{Q}$ is the composition of the Hurewicz map $\omega: \pi_{q}\left(X_{(r)}, *\right) \rightarrow H_{q} S\left(X_{(r)}\right)$ with the map $H_{q} S\left(X_{(r)}\right) \rightarrow$ $H_{q} S\left(X_{(r)}, Q\right)$ induced by the inclusion $Z \rightarrow Q$. By (32) we can always find $[c] \in H_{q} M\left(X_{(r)}\right)$ such that $H_{q}(i)[c]=\omega\left(\left[\sum_{j=1}^{\infty}\left\{f_{j}, g_{j}\right\}\right]\right)$ and hence we conclude that $H_{q} M\left(X_{(r)}\right)$ is uncountable. If $\Omega: H_{q} M\left(X_{(r)}\right) \rightarrow$ $H_{q} M\left(X_{(r)}, Q\right)$ is the map induced by the inclusion $Z \rightarrow Q$, then we have

$$
H_{q}\left(i \otimes \mathrm{id}_{Q}\right) \circ \Omega S([c])=\omega_{Q}\left(\left[\sum_{j=1}^{\infty}\left\{f_{j}, g_{j}\right\}\right]\right)
$$

and hence $H_{q} M\left(X_{(r)}, Q\right)$ is uncountable.

3. By a co-product on a space $\left(X, x_{0}\right)$ we will mean any continuous base point preserving map $\phi:\left(X, x_{0}\right) \rightarrow\left(X \vee X,\left(x_{0}, x_{0}\right)\right)$. In particular we are not assuming $x_{0}$ is a co-identity for $\phi$. For any space $\left(Y, y_{0}\right)$ we can then use $\phi$ to obtain a binary operation on $\left[\left(X, x_{0}\right),\left(Y, y_{0}\right)\right]$ in the obvious way. We will denote the constant map $X \rightarrow y_{0}$ by $\bar{y}_{0}$.

(39) LEMMA. Let $\left(X, x_{0}\right)$ and $\left(Y, y_{0}\right)$ be any spaces and $\phi$ a co-product on $X$. Let $\mu$ be the binary product induced on $\left[\left(X, x_{0}\right),\left(Y, y_{0}\right)\right]$ by $\phi$. Then, for any $c \in S_{q}(X)$ we have:

(40) $S_{q}(\bar{\mu}(f, g))(c)-S_{q}\left(\bar{\mu}\left(f, \bar{y}_{0}\right)\right)(c)$

$$
-S_{q}\left(\bar{\mu}\left(\bar{y}_{0}, g\right)\right)(c)+S_{q}\left(\bar{\mu}\left(\bar{y}_{0}, \bar{y}_{0}\right)\right)(c) \in M_{q} Y .
$$

Proof. Let $\sigma: \Delta_{q} \rightarrow X$ be any simplex. We will show (41) $\bar{\mu}(f, g) \circ \sigma-\bar{\mu}\left(f, \bar{y}_{0}\right) \circ \sigma-\bar{\mu}\left(\bar{y}_{0}, g\right) \circ \sigma+\bar{\mu}\left(\bar{y}_{0}, y_{0}\right) \circ \sigma \in M_{q}(Y)$. From (41) we deduce (40) immediately from the definition of $S_{q}$ and linearity.

To see that (41) holds, let $t \in \Delta_{q}$ and suppose in the first case that $\phi(\sigma(t))=\left(x, x_{0}\right) \in X \vee X$. Then we have

$$
\begin{aligned}
\bar{\mu}(f, g) \circ \sigma(t) & =\Delta \circ(f \vee g) \circ \phi \circ \sigma(t) \\
& =\Delta \circ(f \vee g)\left(x, x_{0}\right)=\Delta\left(f(x), y_{0}\right)=f(x) .
\end{aligned}
$$


A similar computation gives:

$$
\begin{gathered}
\bar{\mu}\left(f, \bar{y}_{0}\right) \circ \sigma(t)=f(x) \\
\bar{\mu}\left(\bar{y}_{0}, g\right) \circ \sigma(t)=y_{0} \\
\bar{\mu}\left(\bar{y}_{0}, y_{0}\right) \circ \sigma(t)=y_{0} .
\end{gathered}
$$

If in the second case $\phi(\sigma(t))=\left(x_{0}, x\right) \in X \vee X$ then we obtain

$$
\begin{aligned}
\bar{\mu}(f, g) \circ \sigma(t) & =g(x) \\
\bar{\mu}\left(f, \bar{y}_{0}\right) \circ \sigma(t) & =y_{0} \\
\bar{\mu}\left(\bar{y}_{0}, g\right) \circ \sigma(g) & =g(x) \\
\bar{\mu}\left(\bar{y}_{0}, \bar{y}_{0}\right) \circ \sigma(t) & =y_{0} .
\end{aligned}
$$

If we let $\left\{v_{i}\right\}$ be the coefficients of the four terms in (41) then we see by (42)-(49) that for any $w \in Y, \Sigma_{I(t, w)} v_{i}=0$. Since $t$ was arbitrary, we conclude by (10) that (41) holds.

(50) Theorem. Let $\left(X, x_{0}\right)$ and $\left(Y, y_{0}\right)$ be any spaces and $\phi a$ co-product on $X$. Let $\mu$ be the binary product induced on $\left[\left(X, x_{0}\right),\left(Y, y_{0}\right)\right]$ by $\phi$. Then, for $q \geq 1$ we have:

(i) if $z \in H_{*} A(X)$ then $\mu(f, g)_{*}(z)=\mu\left(f, \bar{y}_{0}\right)_{*}(z)+\mu\left(\bar{y}_{0}, g\right)_{*}(z)$ and

(ii) if $\alpha \in H^{*} A(X)$ then $\mu(f, g)^{*}(\alpha)=\mu\left(f, \bar{y}_{0}\right)^{*}(\alpha)+\mu\left(\bar{y}_{0}, g\right)^{*}(\alpha)$.

Proof. Suppose $z=[\rho(c)]$ for some $c \in S_{q}(X)$. Then

$$
\begin{aligned}
& \mu(f, g)_{q}(z)-\mu\left(f, \bar{y}_{0}\right)_{q}(z)-\mu\left(\bar{y}_{0}, g\right)_{q}(z) \\
& \quad=\mu(f, g)_{q}(z)-\mu\left(f, \bar{y}_{0}\right)_{q}(z)-\mu\left(\bar{y}_{0}, g\right)_{q}(z)+\mu\left(\bar{y}_{0}, \bar{y}_{0}\right)_{q}(z) .
\end{aligned}
$$

This is because $\mu\left(\bar{y}_{0}, \bar{y}_{0}\right)_{q}(z) \in H_{q} A\left(y_{0}\right)$ and $H_{q} A\left(y_{0}\right)=0$ for $q \geq 1$. Now if we use the fact that for any map $h: X \rightarrow Y, h_{q}[\rho(c)]=$ $\left[\rho\left(S_{q}(h)(c)\right)\right]$, then $(51)$ becomes:

$$
\begin{aligned}
& {\left[\rho\left(S_{q}(\bar{\mu}(f, g))(c)\right)\right]-\left[\rho\left(S_{q}\left(\mu\left(g, \bar{y}_{0}\right)\right)(c)\right)\right]} \\
& -\left[\rho\left(S_{q}\left(\bar{\mu}\left(\bar{y}_{0}, g\right)\right)(c)\right)\right]+\left[\rho\left(S_{q}\left(\bar{\mu}\left(\bar{y}_{0}, \bar{y}_{0}\right)\right)(c)\right)\right]=0 \quad \text { by }(40) .
\end{aligned}
$$

Therefore (i) of (50) holds.

Part (ii) follows in a similar manner.

(52) Corollary. Let $\left(X, x_{0}\right)$ be any space and $\phi$ a co-product on $X$. Suppose $\left(Y, y_{0}\right)$ has the homotopy type of a possibly infinite simplicial 
complex. Let $\mu$ be the binary product induced on $\left[\left(X, x_{0}\right),\left(Y, y_{0}\right)\right]$ by $\phi$. Then, for all $q \geq 1$, we have:

(i) If $z \in H_{q} S(X)$, then $\mu(f, g)_{q}(z)=\mu\left(f, \bar{y}_{0}\right)_{q}(z)+\mu\left(\bar{y}_{0}, g\right)_{q}(z)$

(ii) If $\alpha \in H^{q} S(Y)$, then $\mu(f, g)^{q}(\alpha)=\mu\left(f, \bar{y}_{0}\right)(\alpha)+\mu\left(\bar{y}_{0}, g\right)^{q}(\alpha)$.

Proof. This follows from (18), its analogue in cohomology and (50).

It can be shown that for each of the elements constructed in [BM] there is a canoniclal way of constructing a space $X$ and a co-product $\phi$ providing a counterexample to Corollary (52) when $X$ is not a simplicial complex.

\section{REFERENCES}

[B] George M. Bergman, Boolean rings of projection maps, J. London Math. Soc., Ser. 24 (1971/72), 593-598.

[BM] M. G. Barratt and John Milnor, An example of anomalous singular theory, Proc. London Math. Soc., 13 (1962), 293-297.

[G] Marvin J. Greenberg, Lectures on Algebraic Topology, Mathematics Lecture Note Series. New York: Benjamin 1967.

[R] William J. Ralph, Thesis, University of Waterloo, Waterloo, Ontario Canada 1982.

[S] Edwin H. Spanier, Algebraic Topology, McGraw-Hill 1966.

[W] George Whitehead, Elements of Homotopy Theory, Graduate texts in mathematics 61.

Received June 4, 1984.

UNIVERSITY OF WESTERN ONTARIO

LONDON, ONTARIO, CANADA 



\title{
PACIFIC JOURNAL OF MATHEMATICS EDITORS
}

\author{
V. S. VARADARAJAN \\ (Managing Editor) \\ University of California \\ Los Angeles, CA 90024 \\ HERBERT ClEMENS \\ University of Utah \\ Salt Lake City, UT 84112 \\ R. FINN \\ Stanford University \\ Stanford, CA 94305
}

\author{
HERMANN FLASCHKA \\ University of Arizona \\ Tucson, AZ 85721 \\ RAMESH A. GANGOLLI \\ University of Washington \\ Seattle, WA 98195 \\ VAUGHAN F. R. JONES \\ University of California \\ Berkeley, CA 94720 \\ ROBION KIRBY \\ University of California \\ Berkeley, CA 94720
}

C. C. MOORE

University of California

Berkeley, CA 94720

H. SAMELSON

Stanford University

Stanford, CA 94305

HAROLD STARK

University of California, San Diego

La Jolla, CA 92093

\section{ASSOCIATE EDITORS}

\author{
R. AREnS \\ E. F. BECKENBACH \\ B. H. NEUMANN \\ F. WOLF \\ K. YOSHIDA \\ (1906-1982)

\section{SUPPORTING INSTITUTIONS}

\begin{abstract}
UNIVERSITY OF ARIZONA
UNIVERSITY OF BRITISH COLUMBIA

UNIVERSITY OF CALIFORNIA

MONTANA STATE UNIVERSITY

UNIVERSITY OF NEVADA, RENO

NEW MEXICO STATE UNIVERSITY

OREGON STATE UNIVERSITY
\end{abstract} \\ CALIFORNIA INSTITUTE OF TECHNOLOGY \\ UNIVERSITY OF OREGON \\ UNIVERSITY OF SOUTHERN CALIFORNIA \\ STANFORD UNIVERSITY \\ UNIVERSITY OF HAWAII \\ UNIVERSITY OF TOKYO \\ UNIVERSITY OF UTAH \\ WASHINGTON STATE UNIVERSITY \\ UNIVERSITY OF WASHINGTON
}

The Supporting Institutions listed above contribute to the cost of publication of this Journal, but they are not owners or publishers and have no responsibility for its content or policies.

Mathematical papers intended for publication in the Pacific Journal of Mathematics should be in typed form or offset-reproduced (not dittoed), double spaced with large margins. Please do not use built up fractions in the text of the manuscript. However, you may use them in the displayed equations. Underline Greek letters in red, German in green, and script in blue. The first paragraph must be capable of being used separately as a synopsis of the entire paper. In particular it should contain no bibliographic references. Please propose a heading for the odd numbered pages of less than 35 characters. Manuscripts, in triplicate, may be sent to any one of the editors. Please classify according to the scheme of Math. Reviews, Index to Vol. 39. Supply name and address of author to whom proofs should be sent. All other communications should be addressed to the managing editor, or Elaine Barth, University of California, Los Angeles, California 90024.

There are page-charges associated with articles appearing in the Pacific Journal of Mathematics. These charges are expected to be paid by the author's University, Government Agency or Company. If the author or authors do not have access to such Institutional support these charges are waived. Single authors will receive 50 free reprints; joint authors will receive a total of 100 free reprints. Additional copies may be obtained at cost in multiples of 50 .

The Pacific Journal of Mathematics is issued monthly as of January 1966. Regular subscription rate: $\$ 190.00$ a year (5 Vols., 10 issues). Special rate: $\$ 95.00$ a year to individual members of supporting institutions.

Subscriptions, orders for numbers issued in the last three calendar years, and changes of address should be sent to Pacific Journal of Mathematics, P.O. Box 969, Carmel Valley, CA 93924, U.S.A. Old back numbers obtainable from Kraus Periodicals Co., Route 100, Millwood, NY 10546.

The Pacific Journal of Mathematics at P.O. Box 969, Carmel Valley, CA 93924 (ISSN 0030-8730) publishes 5 volumes per year. Application to mail at Second-class postage rates is pending at Carmel Valley, California, and additional mailing offices. Postmaster: send address changes to Pacific Journal of Mathematics, P.O. Box 969, Carmel Valley, CA 93924.

PUBLISHED BY PACIFIC JOURNAL OF MATHEMATICS, A NON-PROFIT CORPORATION

Copyright (C) 1986 by Pacific Journal of Mathematics 


\section{Pacific Journal of Mathematics}

\section{Vol. 123, No. $2 \quad$ April, 1986}

David Jay Anick, A loop space whose homology has torsion of all orders . . 257 Steven P. Diaz, Space curves that intersect often ................. 263

Thierry Fack and Hideki Kosaki, Generalized $s$-numbers of $\tau$-measurable

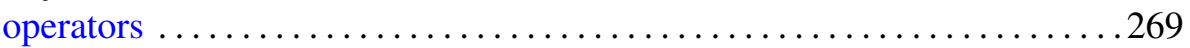

Karl Heinrich Hofmann and Karl Strambach, Lie's fundamental

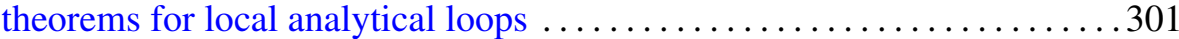

James Secord Howland, On the Kato-Rosenblum theorem ............. 329

Frieder Knüppel and Edzard Salow, Plane elliptic geometry over rings . . . . 337

Alan Noell, Peak points in boundaries not of finite type ................ 385

William J. Ralph, An extension of singular homology to Banach

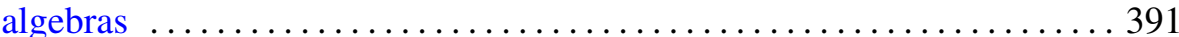

Wade C. Ramey, Averaging properties of pluriharmonic boundary values . . 407

Thomas Joseph Ransford, On the range of an analytic multivalued

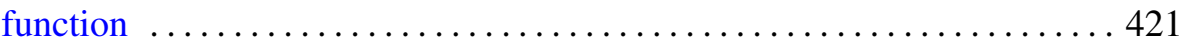

Christopher Donald Sogge, On restriction theorems of maximal-type . . . . 441

Edwin Spanier, Cohomology with supports $\ldots \ldots \ldots \ldots \ldots \ldots \ldots \ldots \ldots 47$

Emil J. Straube, Orthogonal projections onto subspaces of the harmonic

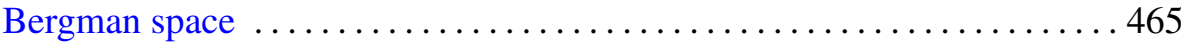

Thomas Vogel, Asymptotic behavior of two semilinear elliptic free boundary problems $\ldots \ldots \ldots \ldots \ldots \ldots \ldots \ldots \ldots$ 